\title{
Role of Deep Brain Stimulation in Treatment Resistant Depression: A Review Article
}

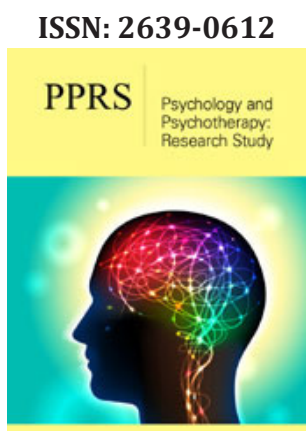

*Corresponding author: Alireza Khoshnevisan, Associate Professor, Tehran University of Medical Sciences, Iran

Submission: 眥 April 19, 2021

Published: 䟧April 30, 2021

Volume 4 - Issue 5

How to cite this article: Sadat Kamali Z Sana Mirahmadi M, Alireza Khoshnevisan. Role of Deep Brain Stimulation in Treatment Resistant Depression: A Review Article. Psychol Psychother Res Stud. 4(5). PPRS. 000598. 2021. 2021. DOI: 10.31031/PPRS.2021.04.000598

Copyright@ Alireza Khoshnevisan, This article is distributed under the terms of the Creative Commons Attribution 4.0 International License, which permits unrestricted use and redistribution provided that the original author and source are credited.

\author{
Sadat Kamali Z ${ }^{1}$, Sana Mirahmadi $\mathbf{M}^{2}$ and Alireza Khoshnevisan ${ }^{3 *}$ \\ ${ }^{1}$ Department of Psychology, Iran \\ ${ }^{2}$ Islamic Azad University of Medical Sciences, Iran \\ ${ }^{3}$ Associate Professor, Tehran University of Medical Sciences, Iran
}

\begin{abstract}
Neuromodulation has been used in Treatment-Resistant Depression (TRD) as a presumed intervention targeting diverse brain regions however, antidepressant properties of DBS for TRD in latest clinical trials are still under debate. We review the main putative neuroanatomical targets of DBS applied for the treatment of intractable TRD in this paper. Different researches imply that stimulation parameters and neuroanatomical locations could influence DBS-related antidepressant effects. Among different neuromodulatory targets that have been studied, DBS in the neuroanatomical structures of the Subcallosal Cingulate Gyrus (SCG), Anterior Limb of Internal Capsule (ALIC), Ventral Capsule/Ventral Septum (VC/VS), Medial Forebrain Bundle (MFB), and Nucleus Accumbans (NAcc) yielded more reliable antidepressant success rates in cases with TRD. Nonetheless, more well-designed randomized double-blind, controlled trials are necessary to entirely evaluate the efficiency, safety and compatibility of these DBS targets for the management of TRD.
\end{abstract}

Keywords: Depression; Neuromudulation; Subsingular gyrus; Medial forebrain bundle

Abbreviations: TRD: Treatment-Resistant Depression; SCG: Subcallosal Cingulate Gyrus; ALIC: Anterior Limb of Internal Capsule; VC/VS: Ventral Capsule/Ventral Septum; MFB: Medial Forebrain Bundle; NAcc: Nucleus Accumbans; DBS: Deep Brain Stimulation; PD: Parkinson's Disease; TRD: Treatment-Resistant Depression; LHb: Lateral Habenula; ITP: Inferior Thalamic Peduncle; TRI: Treatment of Intractable; ACC: Anterior Cingulate Cortex; RSE: Retrosplenial Cingulate Cortex; AC: Anterior Commissure

\section{Introduction}

Treatment-resistant depression is a relentlessly disabling disease with no confirmed treatment choices once several drugs, psychotherapy, and electroconvulsive therapy have been unsuccessful. Even though neuromodulation (Deep Brain Stimulation) (DBS) is an conventional treatment option for Parkinson's Disease (PD), essential tremor and movement disorders, and has been used in Treatment-Resistant Depression (TRD) as a suggested intervention targeting various brain structures, antidepressant effects of DBS for TRD in latest clinical trials are still under debate. In this paper, we review main neuroanatomical targets of DBS proposed for the treatment of unresponsive TRD to verify which brain target(s) have the most successful results regarding acute and maintenance management of TRD. On the whole we considered seven distinct DBS targets that is: Subcallosal Cingulate Gyrus (SCG), Nucleus Accumbens (NAc), ventral capsule/ventral striatum, Anterior Limb of Internal Capsule (ALIC), Medial Forebrain Bundle (MFB), Lateral Habenula (LHb) and Inferior Thalamic Peduncle (ITP) for the Treatment of Intractable TRD. Electrical stimulation of these relevant brain frameworks revealed impacts on mood alteration in cases with TRD.

\section{Cingulate gyrus}

Cingulate gyrus represents an arcuated anatomical structure, which is settled in the medial part of the cerebrum [1], and it encircles the corpus callosum by a demarcating region called the callosal fissure. It emerges underneath the rostrum, arches over the anterior surface of the genu, and broadens alongside the dorsal surface of the trunk, and eventually joins to the hippocampal gyrus via narrow isthmus after terminating to the posterior surface of the splenium [2]. Concerning cytoarchitectural features, the cingulate gyrus comprises of following portions: Anterior Cingulate Cortex (ACC) and Subcallosal Cingulate Gyrus (SCG) as a subset of the anterior cingulate gyrus which is verticalized to the corpus callosum 
encompassing the 32, 25 and 24 Brodmann areas [1], posterior cingulate cortex and Retrosplenial Cingulate Cortex (RSE) [3].

The SCG could affect the pathophysiology of some neural dependent process leading to major depression like cognitive mechanism, mood condition, emotional interactions, reward process, cortisol modulations, circadian rhythm, appetite status ,and the processing of the pain, by synaptically connecting whether with subcortical or cortical structures involving in aforementioned aspects [4]. Herein SCG might play a crucial role in modulating the unpleasant emotions presenting whether in healthy or depressed individuals [5,6]. Here, we will review some of the most recent human research which has been done to explore the effects of SCG-DBS in Treatment Resistant Depressed (TRD) patients. The first double-blind randomized sham-controlled study conducted by Puigdemont and his co-workers [7] and elucidated that DBS (continuous electrical therapy) of SCG might represent a reliable and fruitful therapy for sustaining therapeutic impacts in those who presented with extreme depression.

In sum, antidepressants impression of DBS has vanished following the discontinuation of the stimulations as we ascertain that clear relapse or exacerbating will be occurred following the withdrawal. Moreover, no side effects such as (paresthesia, headache, GI discomfort, dizziness) or serious adverse neuropsychiatric manifestations attributing to the stimulation were observed in the course of the exploration. A randomized sham-controlled trial by the leadership of Holtzhimer affirmed the achievability and safety of SCG-DBS as a novel therapeutic agent for TRD [8]. Albeit in the present study, it is not well established that active stimulation is significantly correlated with antidepressant effects. They bounded the participants up in following the steadily scheduled psychotherapies meeting as well as the sustained medication regimen. Neither limitation of psychotherapy nor alteration of medications was permitted. By evaluating the use of medications, they demonstrated that there is no link between proportions of whom gaining a response. During the twelve months, Participants have faced 40 dramatically adverse events. In this regard overall 8 serious adverse events were recognized to be associated with the operation or study device including one post-Surgical seizure a dermal erosion following the extension wires and six infections. Consequently, assessments revealed more improvement in depression happened after twelve-Months in those who were monitored for twenty-four months.

In a similar study, Merki [9] and his colleagues enrolled Participants to delayed and non-delayed-onset population (shamDBS VS active DBS). Their attempts illustrated that SCG-DBS could be regarded as a safe and long-term beneficial approach for the diminishing of depressive manifestations. Over twenty- four month of monitoring a few proportions of participants responded to SCG-DBS and experienced remission until twenty-eight and forty-eight months later. Interestingly after twenty-four months, just one person presented with limited response. Supportive treatment and antidepressant were prescribed for all of the individuals during the examination and the novel psychotherapy courses were recommended for three patients after 6 months. For all those who obtained concomitant mediation therapy dosage remained constant during stimulation. They figured out that DBS represents a favorable therapy for depression also antidepressant agents affected the reported changes. The sore throat owing to anesthesia, headaches, dizziness, tingling, and pain of the scalp resulted by surgery were the most common adverse events in this trial, nevertheless, no serious catastrophic events of SCG-DBS were reported. In this respect Minor side effects happened mainly due to the battery changes or setting and were readily modified by changes in the settings.

Comparison between high-frequency SCG Stimulation and low-frequency in the patient who presented with TRD was performed by Eitan [10] and his co-workers. After 6 months highfrequency stimulation demonstrated better results in comparison with the low-frequency stimulation. The long term impact of high- frequency Stimulation was accompanied by more favorable outcome (second six months VS first 6 months). All participants were permitted to sustain both arranged scheduled psychotherapy and antidepressant therapeutic procedures. The patients were not given the new drug as well as antidepressant drugs. Just one of the 40 reported unfavourable events was severe. Twenty-eight adverse manifestations were observed as being probably or undoubtedly associated with the device or the procedure, thirteen manifestations occurred before activating the stimulation and 15 occurred during activation of the stimulation without any distinction between low and high-frequency stimulations population.

Crowell [11] and his co-workers performed a long-term monitoring program for four to eight years to assess the impact of DBS on Subcallosal Cingulate Gyrus (SCC) as an assistant therapy for the patient who represented the TRD. No changes of medication were allowed to the patients for the initial 6 months of long-term stimulation. The outcomes reflected safety of DBS and elucidated stable well-founded effectiveness of SCC-DBS for managing the TRD after eight years. Nine patients tried to diminish or terminate the therapeutic schedule \& two patients quitted all of the psychiatric order satisfactorily. Nevertheless, most of those who tried to terminate the therapeutic schedule could not bear the whole giving up. They kept one or more antidepressant treatments on. No adverse manifestations were allocated to any of the stimulation.

A large and growing body of literature verified the efficiency of DBS in managing TRD. Albeit some investigations report no unfavorable effects, other research indicated minimal or device/ procedure surgery-associated adverse effects. Regarding the following up period the outcomes demonstrated that SCG-DBS could be considered as an efficient and ensuring surgical procedure for decreasing depressive manifestation of TRD.it has not well established that whether sustained effectiveness of this process is forecasted on brain milieu after the beginning of the stimulation, or the process of SCG-DBS intrinsically needs adjunctive medical therapy. 


\section{Nucleus Accumbens (NAcc)}

NAcc is regarded as a crucial section of ventral striatum settling on an extended part of basal ProsencePhalon with a close approximation to the Anterior Commissure (AC) [12]. The anterior limb of the internal Capsule accommodates the NAcc in an area between the cortex of piriform/claustrum medially and the vertical limb of the diagonal band of Broca laterally. NAcc also is expanded dorsolaterally and dorsomedially to the ventral part of the putamen and caudate receptively [13]. It is subclassified into a core in the medial position and a shell in the lateral position according to chemical features in this respect, the shell is associated with the limbic nucleus and the core is related to the motor part of the extrapyramidal nucleus [14]. NAcc as the constituent of the limbic system and a vital section of the circuits of the prefrontocortico-striato-pallidal axis, is believed to serve like a limbicmotor connecting pathway and is engaged in some psychomotor cognitive-emotional de performance which has changed in several psychopathologies. Owing accommodating NAcc in subcortical Telencephalon it can play a significant role that leads to depression or another frequent neuropsychiatric disorders [15]. It is known that the aforementioned nucleus possesses dopamine as a major neurotransmitter by which it is counted as a vital center for involving in the experience of pleasure \& reward representing a novel psychosurgical approach [16].

The latest studies illustrated the efficacy of NAcc-DBS in those who presented with TRD. During twelve months longitudinally impacts of NAcc-DBS in the depressive manifestations of patients suffering from TRD \& Cognitive performance were surveyed by Grubert [17] and his Co-workers. In their studies, although withdrawal of pharmacological medication was favorable, it was not practically feasible, however, for most of them treatment was held constantly. The outcome demonstrated no significant evidence of negative outcome in any cognitive examination after twelve months period of NAcc-DBS. By making a comparison with baseline after twelve months surprisingly the patients displayed notable cognitive progression. Owing to general progression of cognition status by NAcc-DBS, it might set off a cognition improving procedure regardless of its antidepressant effects.no unfavorable impacts were mentioned in the investigation.

Bewernick [18] and his colleagues elucidated the longlasting impacts of DBS on NAcc in a population of 11 individuals representing TRD. The Patients were committed to following a stable mediation regimen for at least six weeks prior to participating in the study. This trial indicated that NAcc_DBS is accompanied by sustained antidepressant effects in all those who presented with Severe TRD. Some Patients were being monitored over four years and the patient's response conditions remained unchanged after approximately eight-twelve months of therapy and response Status Continued stably over the next four years. Terrible effects were either associated with the surgical complications (Pain, Swollen eyes, dysphagia) directly related to Parameter change including: Sweating, erythema subjective transient incrementing in tension or anxiety, lasting from few minutes to several hours, or have no relation with DBS-therapy including leg fracture, gastritis, and herniated disc. All of the complications directly linked to DBS treatment are accompanied by transient or stoppable characteristics via Parameter change. Consequently, no Patients reflected any persistent adverse manifestations. In another investigation, Milet [9] and his colleagues announced that stimulation of NAcc (in comparison with caudate nucleus), is accompanying by a better response when high-frequency DBS is used, NAcc DBS leads to a significant enhancement in mood status for 75 Percent of Patients by fifteen months after the beginning of the stimulations. No frequent adverse effects were seen neither during surgical procedure nor in Postoperative time albeit one patient tried to suicide in the first fifteen months of monitoring. They permitted using anti-depressant drugs for those who have had no unstable or severe medical Status.

Tronnier et al. [19] Performed a case study to examine the impacts of DBS on TRD as the main target of this intervention and obesity as the secondary target. The outcomes demonstrated the impacts of DBS of NAcc in Pathological obesity. By employing DBS, the Patient was capable to overcome the desire to eating, reducing the depression, improving the mood condition, feeling more favorable quality of life, and less tendency to binge eating habits. Trouble in falling asleep was the only serious side effect during the investigation which was able to be change by using another stimulation parameter. Accordingly, it is assumed that targeting nucleus accumbens by NAcc in TRD is effective and sustained. Nearly in all of them, no serious/persistent adverse effect was reported which means they were stoppable/transient via changing stimulation Parameters.

\section{Medial Forebrain Bundle (MFB)}

MFB passes through the medial part of the midbrain prosencephalon Zones, particularly the nucleus of the ventral striatum such as NAcc and media frontal cortex (MFC) [20]. A circumstantial anatomical representation of MFB has demonstrated converging Projection fibers to the anterior thalamocortical radiation communicated with Prefrontal Cortex (PFC) as well as anticipated subcortical connections to the ventral Striatal nuclei e.g., NAcc, which are extended directly upwards from anterior limb of internal capsule (ALIC) [21]. MFB regards as a significant mammalian brain construction, responsible for inspiration, reward, and motivation [22,23]. It is significantly correlated with mood disorders like depression owing to possessing a crucial role in the regulation of the human reward system [24]. Reward network including of the amygdala, some nucleus of the hypothalamus, NAcc, ventral tegmentum (also known as Ventral Tegmental Area VTA) are connected by the MFB. Association of dysfunction of aforementioned constructions with depression is reported frequently. Stimulation of the MFB lessens anhedonia in those who suffer TRD by modifying dysfunctional reward network [25]. In this regard, we will assess the impact of DBS-MFB in TRD. The efficacy of the MFB-DBS in four TRD cases evaluated by Fenoy and his colleagues in 2016 as a cohort trial lasting for 52 weeks. Patients were asked to keep their prescription for six weeks prior to and 
after the operation and medication remained continually as much as possible.

They concluded that MFB-DBS results a quick antidepressant impact as affirmed in three patients for the next week of stimulation. In those who were monitored, the impacts remained constant over not less than twenty-six weeks. Vertical diplopia (the most frequent unfavorable event), observed in all participants. In this cohort, just one patient completed fifty-two weeks [26]. Fenoy and his colleagues also conducted a longitudinal study with larger samples two years later to intricate on the constant antidepressant impact of MFB-DBS from their last research. The patients were asked to keep their previous medications for six weeks prior to and after the operation and the treatment remained unchanged as much as possible in all participants. The outcomes supported their initial results, showing that MFB-DBS imposes a quick antidepressant impact. Over 26 weeks and sustained by one year, 80 percent success rate from baseline was seen in the Patients. Vertical diplopia introduces as the most frequent event in all participants accompanying with special contact use and enormous parameter changes that was Transitory and diminished with switching to other settings.

Bewemick [27] and his colleagues investigated the antidepressant impacts of DBS on the superolateral subdivision of the MFB during four years. The outcomes verified the long-lasting antidepressant impact of bilateral DBS to the SIMFB (superolateral branch of the medial forebrain bundle) in those who presented with severe TRD, and Powerful antidepressant impacts were kept up till four years with a handful of side effects. No dramatically adverse effects associated with DBS were reported; the habitually Presenting Strabismus may be restrained via stimulation Parameter change. All Participants were permitted to consume their medications all through the course of the Investigation. Throughout the observation time of the first year, three Patients underwent medications change owing to their recovery in depression.

Coenen [28] and his colleagues performed an RCT with a shamcontrolled group to evaluate the efficiency and security of DBS of SLMFB in the long course. They observed a significant increment in the quality of the patient's life, as well as antidepressant efficiency of DBS and improving Global Assessment of functioning (GAFscale). Acute post-surgical antidepressant impacts without any stimulation also studied, probably as a reaction to the insertion of an electrode, which could due to favorable response or placebo impact. Moreover, long-lasting effect of the antidepressant impact of DBS of SIMFB during at least four years was established. Patients received constant Pre/Post -Surgical medication, for a minimum of eight weeks following the operation. Transient strabismus was the most common adverse effect. Besides, no obvious serious side effect was reported. The consequence of DBS cessation following a long-lasting therapy in psychiatric Patients is poorly known.

Kilian [29] and his collaborates described five depressed Patients who were treated via SIMFB_DBS and also had received interrupted stimulation either accidentally (in those who faced with abrupt battery depletion, wrong usage of or dysfunction of recharging device) or because of deliberate discontinuation of the stimulation. A significant reappearance of depression was detected in all cases throughout days, however depressive manifestations diminished after resuming the stimulation. The outcomes indicated that constant DBS of SIMFB is imperative in TRD for keeping up the response because of transient changes in the function of the brain Following a DBS. Generally speaking, MFB DBS is considered a promising therapeutic target for managing the TRD. Nonetheless, the long-run impact of DBS following stimulation termination is not well established yet and further investigation is required to discover the stability of the impacts. Strabismus and diplopia are regarded as the main adverse effect and both of them were diminished, following the change in setting parameters.

\section{Ventral Capsule/Ventral Striatum (VC/VS)}

The VC/VS target is an undistinguishable anatomical Structure however it comprises the shell of the nucleus accumbens and white matter of caudo Ventral Portion of the anterior part of the internal capsule. The tracts(fasciculus) of the aforementioned zone communicate Ventromedial Prefrontal Cortex (VmPFC), Dorsal Anterior Cingulate Cortex (DACC), Dorsal Prefrontal Cortex (DPFc), Orbitofrontal Cortex (OFC), predominantly medial pulvinar, mediodorsal, midline-infra-laminar, and anterior medial nucleus of thalami, brainstem( Pedunclopontine tegmental nucleus, Ventral tegmental region, Substantia nigra, raphe nucleus), hypothalamus and amygdala [30-32]. The VS includes: nucleus accumbens, olfactory tubercle, anterior perforated Substance, and medioventral Part of the caudate nucleus. Human's fMRI investigations revealed that, VS has Sensitivity to a broad range of reward features. It also evinced incremented activity in response to photograph of food [33], arousing sexual imagery [34], an image of provoking material objects [35], and following signs associated with using alcohol [36]. Here, the reports regarding the effects of VC/VS DBD will be discussed.

Malone [37] and his colleagues observed long-term results of VC/VS DBS in TRD. All cases attained Continuous stimulation as well as follow up for at least six months and a maximum of more than four years. They received a constant regimen of Psychotropic drugs for a minimum period of six weeks before the patient's entrance to the study. Noticeable adverse events were categorized into three subgroups: first, those associated with DBS therapy (e.g., stimulation, operational Procedures, and devices); second: unrelated to DBS (such as underlying disorders) and third: unknown. Elevated depression with/or suicide attempts (associated with cessation of stimulation owing to depletion of Patient's neurostimulator battery or unintentionally deactivation), hypomania, (remitting via modification of stimulation parameters), and insomnia were the most frequent non-life-threatening adverse events. Outcomes proposed that VC/VS -DBS might be useful in patients with highly treatment-refractory depression and decreases depressive manifestation during DBS therapy. Regarding the mean Montgomery-Asberg depression Rating Scale (MADRS) and Hamilton Depression Rating Scale (HDRS), they claimed that scores were decreased approximately 52.6 percent over three months, 47 percent over six months, 45.7 percent over twelve months, and finally 56.4 percent by last monitoring. 
Dougherty and his co-workers accomplished double-blind RCT of ventral capsule /ventral striatum DBS as a promising therapeutic target of TRD by sixteen weeks, in open-label continuation trial [38]. The outcome did not reveal a significant difference between the control and experimental groups. Nevertheless, they discovered that there exists some advance for the cohort over the long term monitoring phase of their trial and 20-26.7 percent of the cases acquired responses in any period over the open-label continuation stage. During sixteen-week blinded-phase, the therapeutic regimens remained unchanged. Every modulation in settings was permitted during the continuation phase for the sake of optimizing therapeutic results. Exacerbating depression, suicidal efforts or suicidal ideation and site-infection following surgical implants were the most frequent serious adverse events. Kubu et all reported neuropsychological result of 25 patients with treatment-resistant major depression (TRD) who took part in a randomized doubleblind trial evaluating active and sham deep brain stimulation (DBS) in the anterior limb of the ventral capsule/ventral striatum (VC/ VS) [39]. Their study showed no considerable difference comparing active versus sham stimulation over time. These data imply that VC/VS DBS in patients with TRD does not considerably affect neuropsychological function.

\section{Ventral Anterior Part of the Internal Capsule (VALIC)}

The Crus anterior or so-called anterior limb of the internal capsule consists primarily of frontopontine fibers, thalamocortical tracts and association fibers between lentiform and caudate nuclei [40]. The thalamocortical radiations are located between frontal cortex and medial nuclei of the thalamus, including a large number of the association fibers between the thalamus and Prefrontal lobe [41]. In particular thalamocortical radiations contain bi-directional Projection fibers among the medial and anterior nuclei of the thalamas and medial Orbitofrontal/frontal gyrus as the constituent of the paleo mammalian cortex (limbic system) [42].

Furthermore, the ALIC communicates widely with the Ventrolateral/dorsolateral prefrontal lobe. The majority of vALIC directly adjoin the caudal Portion of NAcc [43]. In this part, the effects of vALIC DBS in TRD will be discussed. Bergfeld [44] and his colleagues evaluated the efficiency of DBS of vALIC in TRD Cases, by assessing active and sham-controlled phase of stimulation. Outcomes denoted that twenty-four percent of patients partially responded to DBS while 40 percent significantly responded to it. They manifested significantly less depressive symptoms by overactive stimulation in comparison with sham Stimulation. The trial of randomized active sham suggested that declining depressive symptoms are independent of placebo effects. Patients abided adverse events satisfactorily, however, some patients remitted by adjustment of settings excluding nocturia and pollakiuria. Albeit some of the adverse events were observed in the patient (like blurred vision, suicidal ideation, transient manifestations of mania, and disturbance of sleeping), the majority of them do not frequently related to DBS. The Medication remained unchanged however Psychiatrists were permitted to taper medication in the case of resolving symptoms. In a different investigation, Bergfeld et al made a comparison between those who experienced DBS of
vALIC and the healthy control group, to assess the effect of DBS on the cognitive status of those who presented with TRD [45]. In both groups, psychomotor function, Visuospatial/vertebral memory, object naming, cognitive flexibility, and attention's condition were examined. Moreover, TRD Patients underwent a randomized double sham/active-controlled trial to assess the efficiency of the stimulations directly. DBS did not possess a prolonged effect on cognitive functioning in these patients, neither negative nor positive effect over Sham, active Stimulation and optimization of DBS Parameters. Medications remained unchanged, however; psychiatrists were permitted to modify medication according to clinical indication. Van der Wal et al announced the monitoring of the cases of their last investigation [44] over twelve months maintenance phase and evaluated the impact of DBS, nearly twenty-four months after DBS implantation, and after the maintenance phase [46]. Patient's medication modified if needed. VALIC DBS for TRD demonstrated sustained efficiency, 24 months after the operation, with stable manifestations after optimization. This result supports DBS as a variable treatment choice for those suffering TRD. The majority of patients tolerated vALIC DBS. The majority of undesirable events were not significantly correlated with the impact of stimulations or surgery.

In one case running down of battery resulted in a sudden rise in depressive symptoms and suicidal attempts leading to admission. It lasted a few months to reobtain a complete response, several weeks after the battery replacement. This incident demonstrated that discontinuation of stimulation may lead to the recurrence of depressive symptoms even following the long-lasted duration of response and this event emphasizes a vital requirement of timely battery replacement. Even though foregoing investigation about vALIC DBS in those presented with TRD indicated the efficacy of the aforementioned surgical approach, our knowledge about this remains. Fortunately, in none of the researches, serious adverse events were observed attributing to the stimulation. Follow-up studies are imperative for evaluating the long-standing consequences of DBS.

\section{Lateral Habenular complex (LHb)}

Habenula (HB) regards as an old anatomical complex settling in the dorsomedial part of the thalami and plays a crucial role in connecting monoaminergic nuclei of brainstem to the forebrain [47]. The Habenular structure settled on both the posterior side of the third ventricle in the dorsal diencephalon. It is comprised of lateral and medial sub-component based on both functional and morphological features and possesses different links with other brain zones [48]. LHb accepts afferent projection fibers by stria medullaris from limbic prosencephalon that is innervated by lateral hypothalamus, cerebral cortex, Portions of the amygdala, and basal ganglia [49]. Fasciculus retroflexus convey efferent of LHb to pars compacta of the substantia nigra, the cholinergic lateroposterior tegmentum and dorsal serotonergic nuclei, dopaminergic ventral tegmental and GABAergic rostromedial tegmental nuclei $[50,51]$.

The $\mathrm{LHb}$ is responsible for an extensive spectrum of pathophysiological responses including reward processing, reward 
error [52], and major depression. It sends some projection fiber to monoaminergic nuclei of the brainstem. Monoamine nuclei are considered to be correlated with the etiology of depression, especially owing to the critical role of the ventral tegmental area and pars compacta of substantia nigra in the dopamine metabolism, locus coeruleus in the metabolism of noradrenaline, and median/ dorsal raphe in the metabolism of Serotonin [53]. Concentration of 5-HT secreted by the raphe nuclei is decreased in depression. Excess activation of the lateral habenula is the main cause. Sartorius et al hypothesized that due to inhibitory effect of high frequency stimulation on lateral habenula, the inhibition of lateral habenula on raphe nuclei is decreased. This cause reduction of pathological alterations of depression due to increased 5 -HT release by raphe nuclei. They believe that lateral habenula could be a helpful new target for BDS in patients with intractable depression [54].

Hoyer and his colleagues reported the result of long-term course of serum BDNF levels in a 64-year-old woman who was treated with DBS of the lateral habenula for intractable major depressive disorder. They explained that according to the neurotrophin theory, decrement of a brain-derived neurotrophic factor (BDNF) has an essential role in pathomechanism of affective disorder, and the treatment-associated increments in peripheral BDNF is related to beneficial efficacy of antidepressant medications and electroconvulsive treatment. They found that a considerable increase in BDNF serum levels after DBS of the lateral habenula and an inverse U-shaped association of depression scores and BDNF levels occur. Their data showed that DBS, like other effective antidepressant therapies, may cause an increase in peripheral BDNF levels [55].

\section{Inferior Thalamic Peduncle (ITP)}

ITP Join the orbitofrontal cortical area to non-Specific thalamic nuclei [56]. To the best of our knowledge, just one study has been conducted assessing the effect of DBS ITP and we will discuss it in the present research. Jimeneze and his fellow members investigated the impacts of DBS on ITP in those who suffered from TRD. Furthermore, the patient has undergone criteria for bulimia and borderline Personality disorders (BPDs). Receiving eight-month stimulation made her depression better, improved significantly her depression score, and normalized her neuropsychological function, with no antidepressant medication. Eight-month later, she enrolled in the double-blind experiment and the stimulation Subsided [57]. Simultaneously, spontaneous fluctuations in her manifestations were reported. Twelve-month later aforementioned fluctuations vanished by resuming stimulation. Monitoring including neuropsychological and psychiatric assessment, every two months, was done during twenty-four months period. No unfavorable effect was reported. Jimeneze published 9 years follow up result of same patient and stated that the procedure is safe and still effective in long term period [58].

\section{Conclusion}

Researches confirm that stimulation parameters and neuroanatomical structures could make an impact on DBS-related antidepressant property, and also suggested that modifying impressions on monoamine neurotransmitters in target structures or interrelated brain networks subsequent to DBS could have a role in the antidepressant effects of DBS. Among different neuromodulatory targets that have been studied, DBS in the neuroanatomical structures of the subcallosal cingulate gyrus (SCG), anterior limb of internal capsule (ALIC), ventral capsule / ventral septum (VC/VS), medial forebrain bundle (MFB), and nucleus accumbans (NAcc) yielded more reliable antidepressant success rates in cases with TRD. A number of unfavourable events might happen during DBS treatment, even though it is doubtful whether some adverse events can be related to DBS therapy. Nonetheless, more well-designed randomized double-blind, controlled trials are necessary to entirely evaluate the efficiency, safety and compatibility of these DBS targets for the management of TRD.

\section{References}

1. Hamani C, Mayberg H, Stone S, Laxton A, Haber S, et al. (2011) The subcallosal cingulate gyrus in the context of major depression. Biol psychiatry 69(4): 301-308.

2. Gray H, Standring S (2008) Gray's anatomy: The anatomical basis of clinical practice. Churchill Livingstone, UK.

3. Vogt BA (1993) Structural organization of cingulate cortex: Areas, neurons, and somatodendritic transmitter receptors. In Neurobiology of cingulate cortex and limbic thalamus. Birkhäuser, Boston, MA, USA, pp. 19-70.

4. Lozano AM, Mayberg HS, Giacobbe P, Hamani C, Craddock RC, et al. (2008) Subcallosal cingulate gyrus deep brain stimulation for treatmentresistant depression. Biol psychiatry 64(6): 461-467.

5. Drevets WC, Savitz J, Trimble M (2008) The subgenual anterior cingulate cortex in mood disorders. CNS spectrums. 13(8): 663-681.

6. Posse RP, Choi KS, Holtzheimer PE, Crowell AL, Garlow SJ, et al. (2018) A connectomic approach for subcallosal cingulate deep brain stimulation surgery: Prospective targeting in treatment-resistant depression. Mol Psychiatry 23(4): 843-849.

7. Puigdemont D, Portella MJ, Egea PR, Molet J, Gironell A, et al. (2015) A randomized double-blind crossover trial of deep brain stimulation of the subcallosal cingulate gyrus in patients with treatment-resistant depression: a pilot study of relapse prevention. J Psychiatry Neurosci 40(4): 224-231.

8. Holtzheimer PE, Husain MM, Lisanby SH, Taylor SF, Whitworth LA, et al. (2017) Subcallosal cingulate deep brain stimulation for treatmentresistant depression: a multisite, randomized, sham-controlled trial. Lancet Psychiatry 4(11): 839-849.

9. Millet B, Jaafari N, Polosan M, Baup N, Giordana B, et al. (2014) Limbic versus cognitive target for deep brain stimulation in treatmentresistant depression: accumbens more promising than caudate. Eur Neuropsychopharmacol 24(8): 1229-1239.

10. Eitan R, Fontaine D, Benoît M, Giordana C, Darmon N, et al. (2018) One year double blind study of high vs low frequency subcallosal cingulate stimulation for depression. J Psychiatr Res 96: 124-134.

11. Crowell AL, Posse RP, Holtzheimer PE, Garlow SJ, Kelley ME, et al. (2019) Long-term outcomes of subcallosal cingulate deep brain stimulation for treatment-resistant depression. Am J Psychiatry 176(11): 949-956.

12. Mai JK, Assheuer JK, Paxinos G (2004) Atlas of the human brain. $\left(2^{\text {nd }}\right.$ edn), Academic Press. San Diego, USA.

13. Sturm V, Lenartz D, Koulousakis A, Treuer H, Herholz K, et al. (2003) The nucleus accumbens: A target for deep brain stimulation in obsessivecompulsive-and anxiety-disorders. J Chem Neuroanat 26(4): 293-299. 
14. Neto LL, Oliveira E, Correia F, Ferreira AG (2008) The human nucleus accumbens: Where is it? A stereotactic, anatomical and magnetic resonance imaging study. Neuromodulation 11(1): 13-22.

15. Groenewegen HJ, Wright CI, Beijer AV (1996) The nucleus accumbens: gateway for limbic structures to reach the motor system?. In Progress in brain research 107: 485-511.

16. Schlaepfer TE, Cohen MX, Frick C, Kosel M, Brodesser D, et al. (2008) Deep brain stimulation to reward circuitry alleviates anhedonia in refractory major depression. Neuropsychopharmacology 33(2): 368377.

17. Grubert C, Hurlemann R, Bewernick BH, Kayser S, Hadrysiewicz B, et al. (2011) Neuropsychological safety of nucleus accumbens deep brain stimulation for major depression: effects of 12-month stimulation. World J Biol Psychiatry 12(7): 516-527.

18. Bewernick BH, Kayser S, Sturm V, Schlaepfer TE (2012) Long-term effects of nucleus accumbens deep brain stimulation in treatment-resistant depression: evidence for sustained efficacy. Neuropsychopharmacology 37(9): 1975-1985.

19. Tronnier VM, Rasche D, Thorns V, Fischer AD, Münte TF, et al. (2018) Massive weight loss following deep brain stimulation of the nucleus accumbens in a depressed woman. Neurocase 24(1): 49-53.

20. Coenen VA, Schlaepfer TE, Maedler B, Panksepp J (2011) Cross-species affective functions of the medial forebrain bundle-Implications for the treatment of affective pain and depression in humans. Neuroscience \& Biobehavioral Reviews 35(9): 1971-1981.

21. Coenen VA, Panksepp J, Hurwitz TA, Urbach H, Mädler B (2012) Human medial forebrain bundle (MFB) and anterior thalamic radiation (ATR): Imaging of two major subcortical pathways and the dynamic balance of opposite affects in understanding depression. J Neuropsychiatry Clin Neurosci 24(2): 223-236.

22. Alcaro A, Huber R, Panksepp J (2007) Behavioral functions of the mesolimbic dopaminergic system: an affective neuroethological perspective. Brain Res Rev 56(2): 283-321.

23. Zacharopoulos G, Lancaster TM, Bracht T, Ihssen N, Maio GR, et al. (2016) A hedonism hub in the human brain. Cerebral Cortex 26(10): 3921-3927.

24. Russo SJ, Nestler EJ (2013) The brain reward circuitry in mood disorders. Nat Rev Neurosci 14(9): 609-625.

25. La Torre D, Della Torre A, Chirchiglia D, Volpentesta G, Guzzi G, et al. (2020) Deep brain stimulation for treatment-resistant depression: a safe and effective option. Expert Rev Neurother 20(5): 449-457.

26. Fenoy AJ, Schulz P, Selvaraj S, Burrows C, Spiker D, et al. (2016) Deep brain stimulation of the medial forebrain bundle: distinctive responses in resistant depression. J Affect Disord 203: 143-151.

27. Bewernick BH, Kayser S, Gippert SM, Switala C, Coenen VA, et al. (2017) Deep brain stimulation to the medial forebrain bundle for depressionlong-term outcomes and a novel data analysis strategy. Brain Stimul 10(3): 664-671.

28. Coenen VA, Bewernick BH, Kayser S, Kilian H, Boström J, et al. (2019) Superolateral medial forebrain bundle deep brain stimulation in major depression: A gateway trial. Neuropsychopharmacology 44(7):12241232.

29. Kilian HM, Meyer DM, Bewernick BH, Spanier S, Coenen VA, et al. (2019) Discontinuation of superolateral medial forebrain bundle deep brain stimulation for treatment-resistant depression leads to critical relapse. Biological psychiatry 85(6): e23-e24.

30. Jbabdi S, Lehman JF, Haber SN, Behrens TE (2013) Human and monkey ventral prefrontal fibers use the same organizational principles to reach their targets: tracing versus tractography. J Neurosci 33(7): 3190-3201.
31. Lehman JF, Greenberg BD, McIntyre CC, Rasmussen SA, Haber SN (2011) Rules ventral prefrontal cortical axons use to reach their targets: implications for diffusion tensor imaging tractography and deep brain stimulation for psychiatric illness. J Neurosci 31(28):10392-10402.

32. Yang JC, Papadimitriou G, Eckbo R, Yeterian EH, Liang L, et al. (2015) Multi-tensor investigation of orbitofrontal cortex tracts affected in subcaudate tractotomy. Brain imaging and behavior 9(2): 342-352.

33. Beaver JD, Lawrence AD, Van Ditzhuijzen J, Davis MH, Woods A, et al. (2006) Individual differences in reward drive predict neural responses to images of food. J Neurosci 26(19): 5160-5166.

34. Walter M, Bermpohl F, Mouras H, Schiltz K, Tempelmann C, et al. (2008) Distinguishing specific sexual and general emotional effects in fMRI-Subcortical and cortical arousal during erotic picture viewing. Neuroimage 40(4): 1482-1494.

35. Erk S, Spitzer M, Wunderlich AP, Galley L, Walter H (2002) Cultural objects modulate reward circuitry. Neuroreport 13(18): 2499-2503.

36. Braus DF, Wrase J, Grüsser S, Hermann D, Ruf M, et al. (2001) Alcoholassociated stimuli activate the ventral striatum in abstinent alcoholics. Journal of neural transmission 108(7): 887-894.

37. Malone DA, Dougherty DD, Rezai AR, Carpenter LL, Friehs GM, et al. (2009) Deep brain stimulation of the ventral capsule/ventral striatum for treatment-resistant depression. Biological psychiatry 65(4): 267275 .

38. Dougherty DD, Rezai AR, Carpenter LL, Howland RH, Bhati MT, et al. (2015) A randomized sham-controlled trial of deep brain stimulation of the ventral capsule/ventral striatum for chronic treatment-resistant depression. Biological psychiatry 78(4): 240-248.

39. Kubera M, Obuchowicz E, Goehler L, Brzeszcz J, Maes M (2011) In animal models, psychosocial stress-induced (neuro) inflammation, apoptosis and reduced neurogenesis are associated to the onset of depression. Prog Neuropsychopharmacol Biol Psychiatry 35(3): 744-759.

40. Chowdhury F, Haque M, Sarkar M, Ara S, Islam M (2010) White fiber dissection of brain; the internal capsule: A cadaveric study. Turkish neurosurgery 20(3): 314-22.

41. Schmahmann JD, Pandya DN (2006) Fiber Pathways of the Brain Oxford University Press. New York, USA.

42. Martin JH (2012) Neuroanatomy Text and Atlas. McGraw Hill Professional, USA.

43. Gutman DA, Holtzheimer PE, Behrens TE, Berg JH, Mayberg HS (2009) A tractography analysis of two deep brain stimulation white matter targets for depression. Biological psychiatry 65(4): 276-282.

44. Bergfeld IO, Mantione M, Hoogendoorn ML, Ruhé HG, Notten P, et al. (2016) Deep brain stimulation of the ventral anterior limb of the internal capsule for treatment-resistant depression: a randomized clinical trial. JAMA psychiatry 73(5): 456-464.

45. Bergfeld IO, Mantione M, Hoogendoorn ML, Ruhe HG, Horst F, et al. (2017) Impact of deep brain stimulation of the ventral anterior limb of the internal capsule on cognition in depression. Psychol Med 47(09): 1647-1658.

46. Vander Wal JM, Bergfeld IO, Lok A, Mantione M, Figee M, et al. (2020) Long-term deep brain stimulation of the ventral anterior limb of the internal capsule for treatment-resistant depression. Journal of Neurology, Neurosurgery \& Psychiatry 91(2): 189-195.

47. Benarroch EE (2015) Habenula: Recently recognized functions and potential clinical relevance. Neurology 85(11): 992-1000.

48. Klemm WR (2004) Habenular and interpeduncularis nuclei: Shared components in multiple-function networks. Medical Science Monitor 10(11): RA261-RA273. 
49. Geisler S, Trimble M (2008) The lateral habenula: no longer neglected. CNS Spectrums 13(6): 484-489.

50. Araki M, McGeer PL, Kimura H (1988) The efferent projections of the rat lateral habenular nucleus revealed by the PHA-L anterograde tracing method. Brain Research 441(1-2): 319-330.

51. Jhou TC, Geisler S, Marinelli M, Degarmo BA, Zahm DS (2009) The mesopontine rostromedial tegmental nucleus: A structure targeted by the lateral habenula that projects to the ventral tegmental area of Tsai and substantia nigra compacta. Journal of Comparative Neurology 513(6): 566-596.

52. Gomita Y, Gallistel CR (1982) Effects of reinforcement-blocking doses of pimozide on neural system driven by rewarding stimulation of the MFB: A 14C-2-deoxyglucose analysis. Pharmacology Biochemistry and Behavior 17(4): 841-845.

53. Schneider TM, Beynon C, Sartorius A, Unterberg AW, Kiening KL (2013) Deep brain stimulation of the lateral habenular complex in treatmentresistant depression: traps and pitfalls of trajectory choice. Operative Neurosurgery 72(2): 184-193.

54. Sartorius A, Kiening KL, Kirsch P, von Gall CC, Haberkorn U, et al. (2010) Remission of major depression under deep brain stimulation of the lateral habenula in a therapy-refractory patient. Biological Psychiatry 67(2): e9-e11.

55. Hoyer C, Kranaster L, Sartorius A, Hellweg R, Gass P (2012) Longterm course of brain-derived neurotrophic factor serum levels in a patient treated with deep brain stimulation of the lateral habenula. Neuropsychobiology 65(3): 147-152.

56. Skinner JE, Lindsley DB (1967) Electrophysiological and behavioral effects of blockade of the nonspecific thalamo-cortical system. Brain Research 6(1): 95-118.

57. Jiménez F, Velasco F, Pascual SR, Hernández JA, Velasco M, et al. (2005) A patient with a resistant major depression disorder treated with deep brain stimulation in the inferior thalamic peduncle. Neurosurgery 57(3): 585-593.

58. Jiménez F, Nicolini H, Lozano AM, Piedimonte F, Salín R, et al. (2013) Electrical stimulation of the inferior thalamic peduncle in the treatment of major depression and obsessive compulsive disorders. World Neurosurg 80: S30-e17.e25. 